\title{
Propuesta de un certificado de defunción para mejorar el registro y reporte de la muerte en el periodo perinatal
}

\author{
Javier C ontreras-Lemus, M.C., (1) Samuel Flores-Huerta, M.C.,1) \\ Jorge Arturo Cardona-Pérez, M.C.(1)
}

\section{Contreras-Lemus J, Flores-Huerta S, Cardona-Pérez JA. \\ Propuesta de un certificado de defunción para mejorar el registro y reporte \\ de la muerte en el periodo perinatal. \\ Salud Publica Mex 2001;43:217-223.}

El texto completo en inglés de este artículo está disponible en: http://www.insp.mx/salud/index.html

\section{Resumen}

Objetivo. Analizar la forma en que se registran los nacimientos y la muerte en el periodo perinatal, en el Instituto Mexicano del Seguro Social (IMSS), y documentar si el registro de la muerte, en este periodo, es adecuado. Material y métodos Entre enero y marzo de 2000, se aplicó una encuesta en las 37 delegaciones del IMSS, para conocer, del año 1999, el total de nacimientos vivos y muer tos, las defunciones ocurridas antes del séptimo día, considerando su edad gestacional y peso al nacimiento. Con estos datos se analizó la mortalidad hebdomadal e infantil y se calcularon las tasas correspondientes, incluyendo o desagregando a los niños con $<1000$ g y $<28$ semanas de gestación. Resulta dos D urante 1999 nacieron vivos en el IMSS 634892 niños cuyo peso y edad gestacional se desconocen, y muertos 3 984 niños con $\geq 28$ semanas de gestación. Antes de la primera semana de vida extrauterina fallecieron 4556 niños, de los cuales 1385 (30.4\%) pesaron $<1000 \mathrm{~g}$ y tuvieron $<28$ semanas de gestación. Al analizar la mortalidad con 0 sin estos niños, hubo un descenso de más de dos puntos de tasa en la mortalidad hebdomadal e infantil. Conclusiones. En este trabajo se muestra que la forma como actualmente se clasifica y reporta la muerte de los niños, en el perio do perinatal, es inadecuada. Se propone crear sistemas de información que permitan conocer el peso y la edad

\author{
Contreras-Lemus J, Flores-Huerta S, \\ CardonaPérez JA. \\ Proposal for a death certificate \\ to improve recording and reporting \\ of perinatal mortality. \\ Salud Publica Mex 2001;43:217-223. \\ The English version of this paper \\ is available at: http://www.insp.mx/salud/index.html
}

(1) Coordinación de Salud Reproductiva y Materno Infantil, Instituto Mexicano del Seguro Social, México.

Fecha de recibido: 27 de abril de 2000 - Fecha de aprobado: 29 de noviembre de 2000

Solicitud de sobretiros: Dr. Javier Contreras Lemus. Coordinación de Salud Reproductiva y Materno Infantil, Instituto Mexicano del Seguro Social. Mier y Pesado 120, colonia del Valle, 03100 México, D.F., México.

Correo electrónico: contreraslemus@starmedia.com 
gestacional de todos los nacimientos, así como un nuevo certificado de defunción para el registro de la muerte, en este periodo, que incluya dicha información; éste sustituiría a los certificados de defunción fetal y general actuales y permitiría elaborar reportes comparables con otros países. El texto completo en inglés de este artículo está disponible en: http://www.insp.mx/salud/index.html

Palabras clave: mortalidad fetal; mortalidad perinatal; mortalidad infantil; certificado de defunción; IMSS (MX)
The English version of this paper is available at: http:// www.insp.mx/salud/index.html

Key words: fetal mortality; perinatal mortality; infant mortality; death certificates; IMSS (MX)
$\mathrm{H}$ asta 1850, en México, el registro de los nacimientos y defunciones se hacía por parte de las autoridades religiosas. El Estado inicia, bajo su responsabilidad, el registro de estos hechos vitales después de 1860, creando para tal propósito la oficina denominada Registro Civil. ${ }^{1}$ Desde entonces, las ciudades y las cabeceras municipales cuentan con estas oficinas, pero se carece de ellas, aun hoy en día, en las poblaciones muy pequeñas y alejadas de los centros urbanos. ${ }^{1,2}$

En 1956, casi un siglo después, México inicia oficialmente la certificación de la muerte ${ }^{1,3-5}$ con la extensión de un certificado de defunción para muerte fetal y otro para muerte general; las agencias funerarias quedaron a cargo de la impresión y la distribución de estos documentos. La certificación de la muerte fue obligatoria sólo ante procesos legales, civiles o penales. Estos certificados estuvieron vigentes hasta 1986, ${ }^{3}$ año en el que para entender el proceso de salud enfermedad se agregó la siguiente información: a) la escolaridad y la ocupación del fallecido; b) si el difunto tenía o no protección por alguna institución de seguridad social; c) el sitio donde ocurrió la defunción -en caso de ser un hospital se anota nombre y ubicación-; d) si el fallecido tuvo o careció de atención médica en su última enfermedad, y e) las causas de la muerte se codifican de acuerdo con una clasificación internacional para propósitos de comparación con otros países. ${ }^{4-7}$

Estos certificados de defunción continúan vigentes y son la fuente de datos para el informe sobre la muerte, ya sea que ocurra in utero (fetal) o después del nacimiento. Sin embargo, como la muerte de los recién nacidos está inversamente relacionada con la edad gestacional y el peso de nacimiento, para categorizar mejor el momento en que fallecen, sea que nazcan muertos o fallezcan después del nacimiento. En la figura 1 se muestran las etapas en las que internacionalmente se clasifican las edades de la vida, etapas que han servido para conocer en cuál de ellas ocurre la muerte de los niños, por lo que a continuación se describen las que interesan para propósitos de este estudio. ${ }^{8}$ La etapa fetal intermedia va de la semana 22 hasta

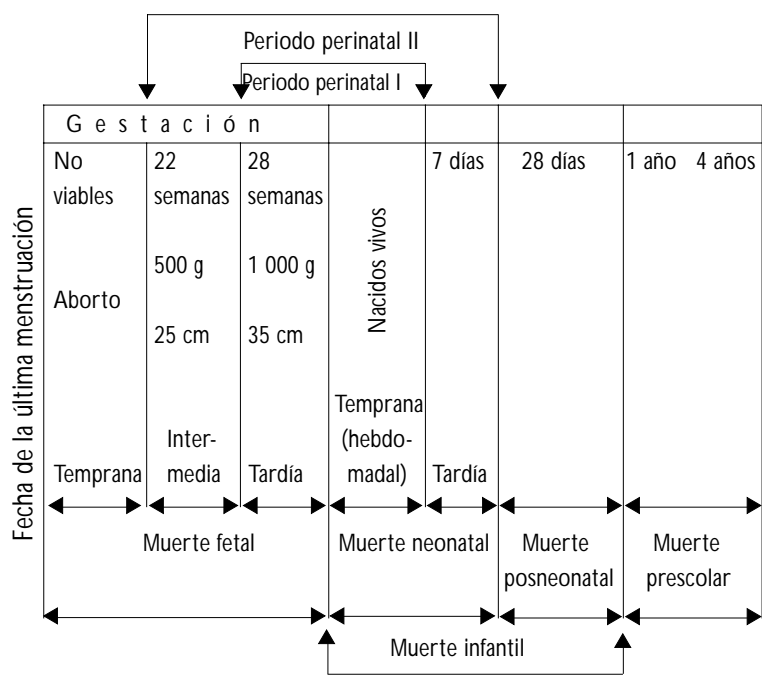

Fuente: referencia 8

Figura 1. Periodos para clasificar los diferentes MOMENTOS EN QUE OCURRE LA MUERTE DE LOS NIÑOS. Instituto Mexicano del Seguro Social, México, eneRO-MARZO, 2000

las $<28$ semanas de gestación; el producto pesa $500 \mathrm{~g}$ o más, pero $<1000$ g, y su longitud es de 25 cm o más, pero $<35 \mathrm{~cm}$. La etapa fetal tardía va de la semana 28 hasta el momento del nacimiento; en esta etapa los fetos pesan $1000 \mathrm{~g}$ o más y su longitud es de $35 \mathrm{~cm}$ o más. Después del nacimiento viene la fase neonatal temprana o hebdomadal, que va del nacimiento hasta $<7$ días, mientras que la fase neonatal tardía va de los 7 días hasta los $<28$ días. Con las etapas fetal tardía y hebdomadal se integra el periodo perinatal I, y con las cuatro etapas descritas se integra el periodo perinatal II. La etapa infantil va del nacimiento hasta antes de cumplirse 365 días de vida. 
Actualmente, la muerte de los niños alrededor del nacimiento se reporta como perinatal I o II y, al interior de cada una de ellas, como fetal intermedia o tardía, neonatal temprana (hebdomadal) o neonatal tardía.

Es importante destacar que las muertes que ocurren en la primera semana de vida representan $55 \%$ de la mortalidad del periodo perinatal $\mathrm{I}^{9}$ y $58 \%$ de las muertes que ocurren durante el primer año de vida,10 por esta razón, ante organismos internacionales, el país adquirió el compromiso de reducirla para el año 2000 en un tercio de la que había en $1990 .{ }^{11}$ No obstante, con el certificado de defunción para muerte general que actualmente se utiliza, no es posible clasificar correctamente la muerte de los niños según las etapas descritas, y como consecuencia, la evaluación de los compromisos adquiridos no tiene la precisión esperada. Por lo tanto, los propósitos de este trabajo son: analizar la forma en que se registran los nacimientos y la muerte en el periodo perinatal en el Instituto Mexicano del Seguro Social (IMSS), documentar si el registro de la muerte de los niños que fallecen en los primeros siete días de vida es adecuado y conocer el impacto de lo que esto representa sobre la mortalidad perinatal, neonatal e infantil. Asimismo, se propone un nuevo certificado de defunción para registrar la muerte de los niños en este periodo.

\section{Material y métodos}

En los meses de enero a marzo de 2000, se aplicó una encuesta en las 37 delegaciones del IMSS para conocer el total de nacimientos vivos y muertos, y defunciones hebdomadales durante el año 1999. De los niños que nacieron vivos y fallecieron antes de los siete días de vida, se solicitó en una cédula o formulario especial, la edad gestacional y peso de nacimiento, debido a que el certificado de defunción para muerte general con el cual se certifica el deceso, no incluye estos datos. Esta información se analizó para conocer la mortalidad hebdomadal e infantil, antes y después de desagregar a los niños con $<1000 \mathrm{~g}$ y $<28$ semanas de gestación.

Puesto que no se conoce el peso al nacimiento y la edad gestacional de los nacidos vivos, para obtener la mortalidad hebdomadal se utilizó la siguiente fórmula: ${ }^{12}$

Nacidos muertos de $\geq 28$ semanas de gestación

Nacidos vivos y fallecidos entre $0 \mathrm{a}<7$ días

Tasa $\frac{\text { de cualquier edad gestacional }}{\begin{array}{c}\text { Nacidos muertos de } \geq 28 \text { semanas de gestación }+ \\ \text { nacidos vivos de cualquier edad gestacional }\end{array}} \times 1000$
La mortalidad infantil se obtuvo de acuerdo con la siguiente fórmula: ${ }^{8}$

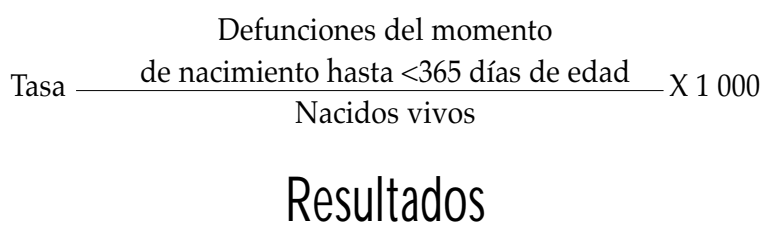

Como se muestra en el cuadro I, en el IMSS, durante 1999, ${ }^{9}$ ocurrieron 641275 nacimientos, de los cuales 634892 nacieron vivos. De éstos no se pudo conocer su edad gestacional y peso.

Los niños que nacieron muertos fueron 3984 , quienes tuvieron $\geq$ de 28 semanas de edad gestacional.

Entre 0 y 7 días de vida extrauterina, fallecieron 4556 niños, de quienes se logró documentar que 1385 pesaron $<1000 \mathrm{~g}$ y tuvieron $<28$ semanas de gestación; se debe mencionar que este número no es el total de los niños con estas características.

Fallecieron 8145 niños de entre 0 y $<365$ días, en el lapso de estudio.

Al desagregar a los niños que pesaron al nacimiento $<1000 \mathrm{~g}$ y tuvieron $<28$ semanas de edad gestacional, la mortalidad perinatal I (fetal tardía y hebdomadal) cambió de una tasa de 13.37 a 11.22; la hebdomadal, de 7.13 a 4.97, y la infantil, de 12.83 a 10.65 . Estos datos y las diferencias entre las tasas de mortalidad se muestran en el cuadro II.

\section{Cuadro I \\ Mortalidad perinatal I, Según Registro. Instituto Mexicano del Seguro Social. Méxıco, 1999}

A. Total de nacimientos $(B+D+E) \quad 641275$

B. Total de nacidos vivos 634892

C. $\mathrm{N}$ acidos vivos (<28 semanas)

D. $N$ acidos muertos (<28 semanas) 2399

E. $N$ acidos muertos ( $\geq 28$ semanas) $\quad 3984$

F. N acimientos (B+E) 638876

G. N eonatos fallecidos de 0 a $<7$ día 4556

H. D efunciones perinatales I (E+G) 8540

I. N eonatos $<28$ semanas nacidos vivos, $y$ fallecidos

de 0 a $<7$ días 1385

\begin{tabular}{lr}
\hline J. Nacimientos (F-I) & 637491
\end{tabular}

K. Fallecidos de 0 a $<365$ días 8145

Fuente: Boletín Estadístico de Mortalidad 1999. Dirección de Prestaciones Médicas, Coordinación de Salud Comunitaria, IMSS 


\section{Cuadro II \\ Mortalidad neonatal temprana, PERINATAL I E INFANTIL RESTANDO LOS NEONATOS CON 28 SEMANAS DE gestación. Instituto Mexicano del Seguro Social. México, 1999}

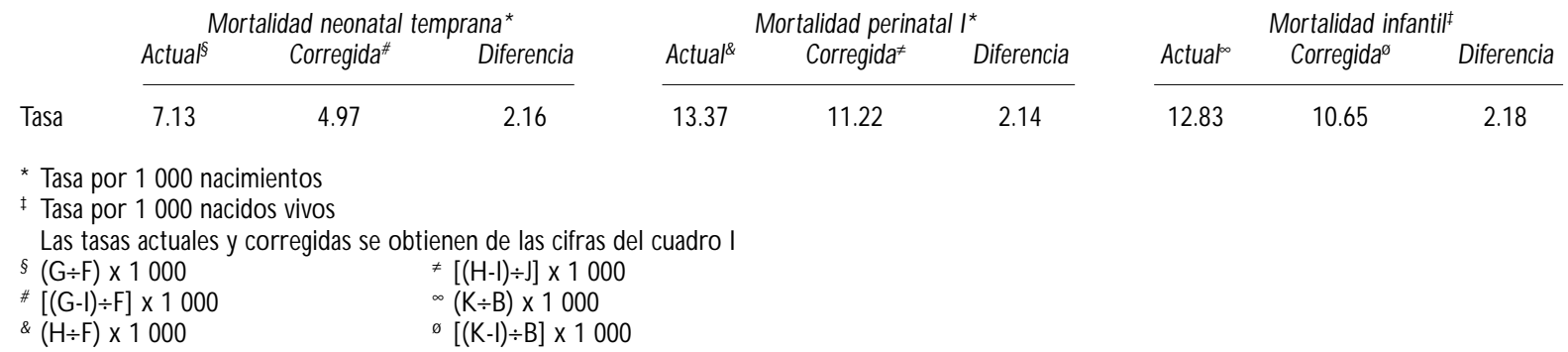

Fuente: Boletín Estadístico de Mortalidad 1999. Dirección de Prestaciones Médicas, Coordinación de Salud Comunitaria, IMSS

\section{Discusión}

Los actuales certificados de defunción tienen la lógica de que uno se usa cuando un niño nace muerto, y otro cuando nace vivo y fallece posteriormente. Sin embargo, cuando la muerte ocurre en el periodo perinatal, que comprende parte de la gestación y los primeros días de la vida extrauterina, y se desean utilizar los datos de estos documentos para reportar indicadores de mortalidad, ${ }^{13-16}$ o para planear servicios de salud, ${ }^{17}$ aparecen vacíos de información; esto se debe fundamentalmente a que la gestación y los primeros días de la vida extrauterina se contemplan como dos momentos desvinculados, en vez de considerarlos estrechamente relacionados. Por otra parte, tampoco se ha considerado que los fallecimientos de la primera semana de vida forman parte del universo que son todos los nacidos vivos. En otras palabras, no se podrá analizar correctamente la mortalidad ${ }^{18} \mathrm{si}$ no se conocen la edad gestacional y el peso al nacer de los niños que nacen vivos y de los niños que fallecen en este periodo.

Con el actual certificado de muerte fetal, que sí contempla la edad gestacional y el peso al nacer, los abortos $\left(<20\right.$ semanas $^{4-6} \mathrm{o}<22$ semanas) ${ }^{7,8}$ y los óbitos ( $\geq$ de 20-22 semanas) ${ }^{4-8}$ se pueden clasificar en las categorías descritas en la figura 1 . Sin embargo, cuando fallecen neonatos, de entre $0 \leq 7$ días, la muerte se reporta utilizando un certificado de defunción de muerte general y lo más que se puede hacer es asignar estos fallecimientos a la mortalidad hebdomadal, ${ }^{18}$ porque, como se ha dicho, este documento no solicita la edad gestacional ni el peso al nacer del niño fallecido. La Organización Mundial de la Salud (OMS) no recomienda incluir en la mortalidad hebdomadal a los niños con peso al nacer $<1000 \mathrm{~g}$ y con edad gestacional $<28$ semanas, porque los neonatos con peso extremadamente bajo alteran la validez de las comparaciones. ${ }^{8,18}$ En efecto, en el presente estudio, 1385 niños (30.4\%) fueron asignados a la mortalidad hebdomadal, cuando en realidad pertenecían a la etapa fetal intermedia (figura 1), y que al desagregarlos, cambiaron las tasas de mortalidad perinatal, hebdomadal e infantil disminuyendo en más de dos puntos de tasa; esto sin considerar que dichos niños no fueron todos los de $<1000 \mathrm{~g}$ y $<28$ semanas de edad gestacional que fallecieron. Es decir, para obtener las tasas de mortalidad, el numerador y el denominador se deberán limitar a fetos y recién nacidos que pesen $\geq 1000 \mathrm{~g}$ (tasa específica por peso), ${ }^{8}$ situación que no ocurre en las condiciones actuales.

Por otra parte, asumiendo que se conoce el número de niños con $<1000 \mathrm{~g}$ y $<28$ semanas que fallecen, se ignora el número de niños de estas características que sobreviven y, por lo tanto, no se pueden elaborar indicadores veraces. Es importante resaltar, que si bien en el nivel donde se manejan las estadísticas existe este vacío de información (edad gestacional y peso al nacer de los nacidos vivos y muertos, así como otras variables maternas), ésta se genera en todos los lugares donde se atienden los nacimientos; así pues, se requiere crear sistemas simplificados de registro y captura para elaborar indicadores y analizar correctamente la mortalidad.

La solución es aparentemente simple: agregar a los certificados de muerte general la edad gestacional y peso al nacimiento, o bien, registrar la muerte que ocurra en el periodo perinatal en un nuevo certificado de defunción que sustituya, para este periodo, a los anteriores. Esta última propuesta se muestra en el 
anexo único; en él se reúne la información relevante de los certificados de muerte fetal y general, y las celdas sombreadas corresponden a las "nuevas" que se proponen; éstas destacan la solicitud de la edad gestacional y el peso al nacer, así como información materna sobre antecedentes obstétricos y evolución del último embarazo. En el apartado de las causas de muerte se recaban datos sobre la patología materna, cuyo análisis informará si está asociada a la defunción del niño.

El certificado propuesto serviría para el informe del deceso de los nacidos muertos y de los fallecidos en el periodo perinatal, y el actual certificado de defunción general, para registrar las muertes que ocurran después de los 28 días de vida.

Desde 1975, la OMS ${ }^{5}$ propone un certificado de muerte perinatal que permita el análisis por separado de la mortalidad en esta etapa; no se encontró información donde se esté aplicando un certificado de defunción para muerte perinatal, pero son numerosos los artículos que refieren la necesidad de modificar la forma de reporte de esta mortalidad, para hacerla comparable entre países, así como los que indican la necesidad de marcar un nuevo límite de viabilidad de los neonatos. ${ }^{18-26}$ En el ámbito nacional se identifican las mismas necesidades, pero además es indispensable corregir el sub y sobrerregistro de la mortalidad fetal y neonatal, y evaluar su impacto en la mortalidad infantil. 2,10,14-16,27-29

Afortunadamente, México cuenta con la normatividad específica en esta área, ${ }^{12,30,31}$ la cual, si se adecua, permitiría avanzar en esta dirección. Colateralmente, se tendría una aproximación entre la patología materna y las causas de muerte de los neonatos.

Como aspecto muy relevante, la información permitiría elaborar indicadores veraces para que no sólo mejore la estadística, sino para planear los servicios de salud ${ }_{1}^{17}$ así como para mejorar los procesos de atención de la salud del binomio madre-hijo, tratando de disminuir el nacimiento de prematuros y evaluando su impacto en la mortalidad infantil. ${ }^{10}$

En conclusión, en este trabajo se muestra cómo una correcta clasificación de la muerte hebdomadal puede disminuir hasta en dos puntos de tasa la mortalidad de esta etapa y la infantil. Asimismo, se propone un certificado para el registro de la muerte para el periodo perinatal que incorpore información relevante del niño, como su edad gestacional y peso al nacer, e información materna asociada a la defunción del homigénito o del infante.

Las modificaciones que se proponen llaman la atención sobre un hecho real que está ocurriendo alre- dedor de la mortalidad perinatal, y se difunden para su análisis por la comunidad médica y de salud.

Si no se avanza en estos cambios, la mortalidad perinatal quedaría atada a conceptos que dieron respuesta a necesidades de dos decenios atrás, sin considerar que también en el presente se tiene la responsabilidad de trabajar para mejorarla.

\section{Referencias}

1. SepúlvedaAJ, López CM, Fernández-de Hoyos RM. Los nuevos modelos de certificados de defunción y de muerte fetal. En:Taller de información para el establecimiento de los nuevos certificados de defunción y muerte fetal en el Distrito Federal. México, D.F.: SSA,1987.

2. Kumate-Rodríguez J. La mortalidad infantil en México. Gac Med Mex 1990;126:475-479.

3. Poder Ejecutivo Federal. Decreto por el que se da a conocer la forma oficial de los certificados de defunción y de muerte fetal. Diario 0 ficial de la Federación, México, D. F., 21 de noviembre de 1986:47-52.

4. W orld $\mathrm{H}$ ealth $\mathrm{O}$ rganization. The prevention of perinatal morbidity and mortality. Report on a seminar. Public Health Papers 1972;42:17-20.

5. O rganización Panamericana de la Salud. Clasificación internacional de enfermedades. Manual de clasificación estadística internacional de enfermedades, traumatismos y causas de defunción (CIE-9). N ovena revisión. México, D.F:: Secretaría de Salud, 1975.

6.W orld Health 0 rganization: Recommended definition, terminology and format for statistical tables related to the perinatal period and use of a new certificate for cause of perinatal deaths. Acta 0 bstet Gynecol Scand 1977;56:247-253.

7. Chiswick ML. Commentary on current World Health O rganization definitions used in perinatal statistics. Arch D is Child 1986;61:708-710. 8. 0 rganización Panamericana de la Salud. Clasificación estadística internacional de enfermedades y problemas relacionados con la salud. 10a. revisión. W ashington, D.C.: O PS, 1995; vol. 2 (Publicación científica núm. 554):161.

9. Instituto Mexicano del Seguro Social. Dirección de Prestaciones Médicas-Coordinación de Salud Comunitaria. Boletín Estadístico Anual de Mortalidad. México, D.F.: IMSS, 1989 a 1999.

10.Vandale S, Rascón-Pacheco RA, Kageyama ML.Time-trends and causes of infant, neonatal and postneonatal mortality in Mexico, 1980-1990. Salud Publica Mex 1997:39:48-52.

11. Cumbre mundial a favor de la infancia. Declaración mundial sobre la supervivencia, la protección y desarrollo del niño.W ashington, D. C.: 0 rganización de las $\mathrm{N}$ aciones Unidas, 1991.

12. Poder Ejecutivo Federal. Acuerdo N 0.127 por el que se crea el Comité $\mathrm{N}$ acional para el Estudio de la Mortalidad Materna y Perinatal. Diario 0 ficial de la Federación, México, D.F., 2 de agosto de 1995:85-87.

13. Instituto $\mathrm{N}$ acional de Estadística, Geografía e Informática. Estadísticas de mortalidad fetal. Boletín de Estadísticas Continuas Demográficas y Sociales. A guascalientes: IN EGI, 1996;1:1-4.

14. Instituto Nacional de Estadística, Geografía e Informática. Dirección Regional C entro. A nálisis sobre la cobertura en la información de la muerte fetal. México, D.F.: IN EGI,1993.

15. Consejo N acional de Población. La situación demográfica de México. México, D.F.: Conapo, 1997:17-20.

16. Secretaría de Salud-Subsecretaría de Prevención y C ontrol de Enfermedades-Dirección General de Estadística e Informática. Mortalidad 1997. México, D.F.: SSA, 1998:73. 
17. López-Cervantes M. Utilización de los datos de mortalidad para la planeación de los servicios de salud: el caso de México. Salud Publica Mex 1985;27:124-139.

18. Perinatal mortality rates. Time for change? Lancet 1991;337:31.

19. Lawson SJ, Mayberry P. H ow can infant and perinatal mortality rates be compared internationally. World Health Forum 1994;15:85-87.

20. Morrison JJ, Rennie MJ. C hanging the definition of perinatal mortality. Lancet 1995;346:1038.

21.W egman ME. Infant mortality: Some international comparisons. Pediatrics 1996;98;1020-1027.

22. MacFarlane A. Attitudes to viability of preterm infants. BMJ 1990; 300:942.

23. Leppert PC. An analysis of the reasons for Japan's low infant mortality rate. J N urse Midwifery 1993;38(6):353-357.

24. Kaushik SL, Parmar VR, G rover N , Kaushik R. N eonatal mortality rate: Relationship to birth weight and gestational age. Indian J Pediatr 1998;65:429-433.

25. Allen MC, D onohue PK, D usman AE. The limit of viability, neonatal outcome of infants born at 22 to 25 weeks' gestation. N Engl J Med 1993; 329:1597-1601.
26. Kaul SS, Babu A, Chopra SRK. Fetal growth from 12 to 26 weeks of gestation. Ann Hum Biol 1986;13:563-570.

27. Tomé P, Reyes H, Piña C, Rodríguez L, Gutiérrez G. Características asociadas al subregistro de muerte en niños del estado de Guerrero, México. Salud Publica Mex 1997;39:523-529.

28. Langer A, Bobadilla JL, Schlaepfer-Pedrazzini L. Limitaciones de la mortalidad infantil como indicador de salud. Salud Publica Mex 1990;32: 467-473.

29. Echeverría-Eguiluz M, Sauri-Pat RA, 0 sorno-C ovarrubias L, A guilarVivas I. Mortalidad perinatal en un hospital regional de tercer nivel. Bol Med Hosp Infant Mex 1994;51:765-769.

30. Poder Ejecutivo Federal. Norma 0 ficial Mexicana N O M-017-SSA21994, para la Vigilancia Epidemiológica. Diario 0 ficial de la Federación. México, D.F., 11 de octubre de 1999:53-80.

31. Poder Ejecutivo Federal. N orma 0 ficial Mexicana N O M-007-SSA21993. Atención a la mujer durante el embarazo, parto y puerperio y del recién nacido. Criterios y procedimientos para la prestación del servicio. Diario 0 ficial de la Federación. México, D.F., 6 de enero de 1995:19-38. 
INSTITUTO MEXICANO DEL SEGURO SOCIAL

COORDINACION DE SALUD REPRODUCTIVA Y MATERNO INFANTIL

PROPUESTA DE CERTIFICADO DE DEFUNCIÓN PERINATAL

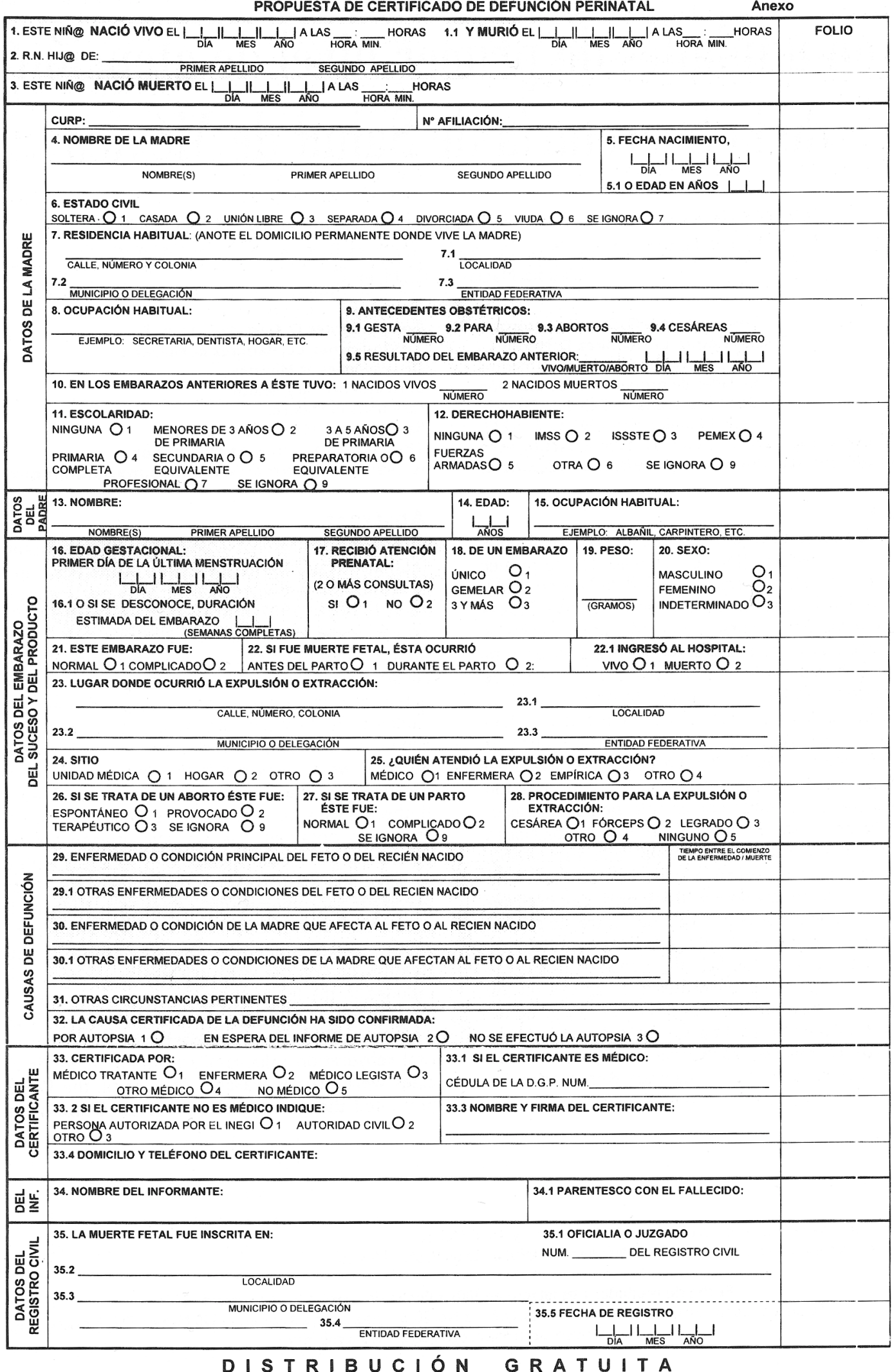

D ISTR I B U C I ÓN GRATU|TA 